\title{
Description of a new species of Chrysina Kirby, 1828 (Coleoptera: Scarabaeidae: Rutelinae) from optima group, based on morphological characters and mtDNA COX I molecular marker
}

urn:Isid:zoobank.org:pub:1F890205-8203-481E-A843-64201288078A

\author{
A.S. Zubov ${ }^{1}$, N.V. Ivshin ${ }^{2}$, A. Yu. Titarenko ${ }^{3}$, D. A. Romanov ${ }^{2}$ \\ ${ }^{1}$ Russia, Moscow, 117556. Bolotnikovskaya 6B, ap.103. \\ E-mail: riprulez@mail.ru \\ ${ }^{2}$ Laboratory of Insects Genetics, Vavilov Institute of General Genetics, \\ Gubkina-3, Moscow, 119333, Russia. \\ E-mail:nikolay.v.ivshin@gmail.com;dromanov16@mail.ru \\ 3 Publicly Traded Company "Morpho Absoloni" \\ E-mail:odindva3@gmail.com
}

\begin{abstract}
A new species of the genus Chrysina Kirby, 1828 is decribed from Panama. Chrysina valentinisp.n. is related to Chrysina tricolor (Ohaus, 1922) and Chrysina optima (H. W. Bates, 1888) in morphology and mtDNA COX I molecular marker. Three related species differ in elytra colour, elytral striae, and number of tubercles on foreleg femur. The morphological divergence of the compared species is supported by distances in mtDNA COI molecular marker.
\end{abstract}

Keywords. Scarabaeidae; Rutelini; Chrysina; Plusiotis; new species; Panama

\section{Introduction}

The genus Chrysina Kirby, 1828 (Coleoptera: Scarabaeidae: Rutelinae) contains about 120 species (Hawks, 2017; Moore et al., 2017; Zubov, Ivshin, Titarenko, Andrianov, 2019) and widespread in the North of C. America (starting from Texas, Usa) to the North-West of South America (Peru). In present study we focused on populations distributed along mountain range Cordillera de Talamanca and a number of species close to Chrysina optima (H. W. Bates, 1888) (Figs 1-4).

C. optima was described from Costa Rica, Cartago (Bates, 1888). The species contains one synonym initially described as Plusiotis optima var. melior (Rothschild and Jordan, 1894) (also from Costa Rica, Cartago), and later synonymized by Morón (1990). There were several discussions about new species found in Panama. Questionable specimens from Panama (Chiriqui) were previously mentioned by Morón (1990) and Ratcliffe (2002), but no new names were given. In this article we compare the new species with C. optima because it appears to be the closest morphologically.

\section{Material and methods}

About 170 specimens from the optima group were available for the study in research collections of the Museum für Naturkunde - Museum für Naturkunde, Leibniz Institut für Evolution und Biodiversitatsforschung (Berlin, Germany), private collection of V.A. Kalinin (Russia, Moscow), and private collection of Andrey Yu. Titarenko (Moscow, Russia). For morphological examination the authors used stereo microscope Zeiss Stemi 2000-C and Canon DSLR with macrolenses and photostacking firmware (Magic Lantern).

Tissue samples (abdomen tergites) were taken from 12 specimens of Chrysina for DNA analysis, and for comparison and estimation of the pairwise distances. 20 specimens from previous study (Zubov, Ivshin, Titarenko, Andrianov, 2019) were added to final dataset for picture completeness and to form the outgroup. The results were deposited in BOLD public project data records - see the Published Projects section of BOLD (Ratnasingham \& Hebert, 2007; www.barcodinglife.org). DNA was extracted with standard phenol-chloroform method (Sambrook, Fritsch, \& Maniatis, 1989). PCR was performed in a T100 thermal cycler (Bio-Rad, USA). Amplification reactions for most samples were carried out according to BOLD protocol with minor improvements in PCR thermal regime algorithm in the final volume of $25 \mu \mathrm{L}$ with $12.5 \mathrm{pmol}$ (or $0.5 \mathrm{pmol} / \mu \mathrm{L}$ ) of the primer LCO1490 (5'-GGTCAACAAATCATAAAGATATTGG-3') and 12.5 pmol (or $0.5 \mathrm{pmol} / \mu \mathrm{L}$ ) of the primer HCO2198 (5'-TAAACTTCAGGGTGACCAAAAAATCA-3') (Folmer, Black, Hoeh, Lutz, \& Vrijenhoek, 1994), 0.1g of the isolated DNA and the universal Encyclo Plus PCR kit (Evrogen, Moscow) following the manufacturer's 
protocol. The PCR thermal regime consisted of an initial denaturation of $4 \min 30 \mathrm{sec}$ at $94^{\circ} \mathrm{C}$; five cycles of 30 sec at $94^{\circ} \mathrm{C}$, $20 \mathrm{sec}$ at $45^{\circ} \mathrm{C}$ and $1 \mathrm{~min}$ at $72^{\circ} \mathrm{C} ; 35 \mathrm{cycles}$ of $30 \mathrm{sec}$ at $94^{\circ} \mathrm{C}, 20 \mathrm{sec}$ at $55^{\circ} \mathrm{C}$ and $1 \mathrm{~min}$ at $72^{\circ} \mathrm{C}$, and a final extension of 5 min at $72^{\circ} \mathrm{C}$. Product size has length $709 \mathrm{bp}$. For some samples we had to apply our original primers and thermal regime to get better results: amplification reactions were carried out in PCR thermal regime algorithm in the final volume of $25 \mu \mathrm{L}$ with $12.5 \mathrm{pmol}$ (or $0.5 \mathrm{pmol} \mu \mathrm{L}^{-1}$ ) of the primer LPX-F (5'- TTTGAGCAGGAATAGTAGGAACTT-3') and 12.5 pmol (or 0.5 pmol $\left.\mu \mathrm{L}^{-1}\right)$ of the primer LPX-R (5'- CCTCCAGCAGGATCAAAA-3'), $0.1 \mathrm{~g}$ of the isolated DNA and the universal Encyclo Plus PCR kit (Evrogen, Moscow) following the manufacturer's protocol. The PCR thermal regime consisted of an initial denaturation of 4 min $30 \mathrm{sec}$ at $94^{\circ} \mathrm{C}$; $37 \mathrm{cycles}$ of $30 \mathrm{sec}$ at $94^{\circ} \mathrm{C}, 20 \mathrm{sec}$ at $57^{\circ} \mathrm{C}$ and $45 \mathrm{sec}$ at $72^{\circ} \mathrm{C}$, and a final extension of $5 \mathrm{~min}$ at $72^{\circ} \mathrm{C}$. Product size has length $604 \mathrm{bp}$. This fragment of COI gene is located inside "barcode" fragment used for BOLD protocol. Amplification success was checked by electrophoresis in $1.5 \%$ agarose gel with subsequent identification of PCR fragments under UV light after staining with ethidium bromide. The section of mtDNA is standard "barcode region" of the Cytochrome Oxidase subunit I gene was sequenced with above primers LCO1490, HCO2198, LPX-F and LPX-R at the Evrogen laboratory (Institute of Bioorganic Chemistry of Russian Academy of Sciences, Moscow). DNA sequences were verified, aligned and analysed using MEGA version 6.0 (Tamura, Stecher, Peterson, Filipski, \& Kumar, 2013). The maximum likelihood method (Tamura-Nei model) was applied for estimating of divergence. For estimating branch support bootstraping was carried out. Bootstrap values above 70 (expressed as percentages of 500 replications) were shown at branch points of the likelihood tree. The new sequences deposited in the BOLD database are shown in Table 1. Sequences from our previous publication (Zubov, Ivshin, Titarenko, Andrianov, 2019) used for comparisons are not included.

Table 1. Specimen data, sequences and images deposited in the BOLD project database (http://www.boldsystems.org).

\begin{tabular}{ll}
\hline \multicolumn{1}{c}{ Taxon name } & BOLD sample Id \\
\hline Chrysina resplendens & BC-Ivsh-6208-73, BC-Ivsh-6208-74 \\
Chrysina optima & BC-Ivsh-6201, BC-Ivsh-6202, BC-Ivsh-6203 \\
Chrysina kalinini & BC-Ivsh-6204-1, BC-Ivsh-6205-2, BC-Ivsh-6206-3, BC-Ivsh-6207-4 \\
Chrysina valentini & BC-Ivsh-6210-2, BC-Ivsh-6210-3, BC-Ivsh-6210-26
\end{tabular}

\section{Abbreviations.}

The following abbreviations are used to indicate the location of the paratype specimens:

MNKB - Museum für Naturkunde, Leibniz Institut für Evolution und Biodiversitatsforschung (Berlin, Germany)

VK - the private collection of V. Kalinin (Moscow, Russia),

AT - the private collection of Andrey Yu. Titarenko (Moscow, Russia),

AZ - the private collection of A.S. Zubov (Moscow, Russia)

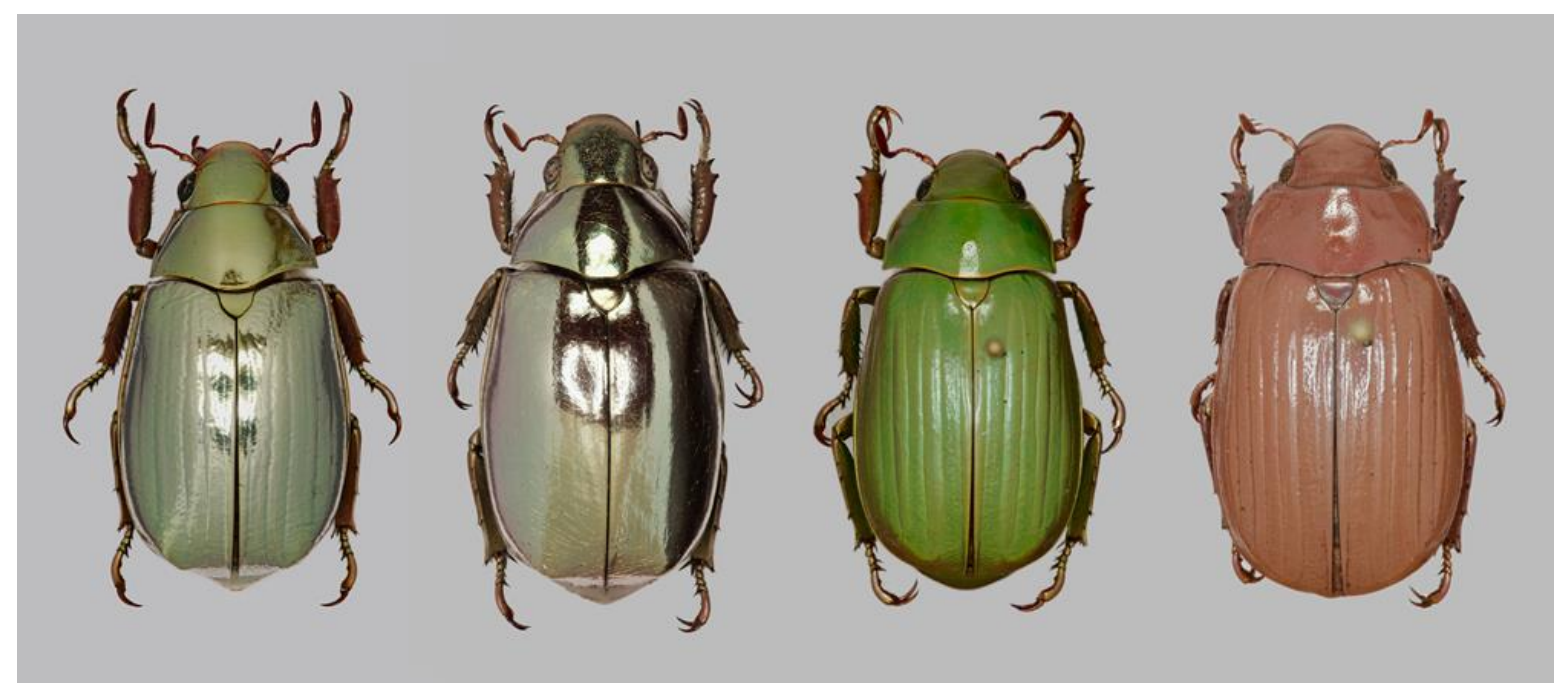

Figs. 1-4. 1. C. valentini sp.n., male, Holotype: dorsal view. Panama, Ngäbe-Buglé, Nole Duima, Jädeberi, 8³1'51"N 8147'55"W, 1683 m, 20.05.2019 (BOLD sample ID BC-Ivsh-6212-26); 2. C. optima, male: dorsal view. Costa Rica, Limon prov., Guacimo, Lomas de Guacimo, Alto la Perla, 1100-2000 m, VI. 2013 (BOLD sample ID BC-Ivsh-6202); 3. C. tricolor, male: dorsal view. Costa Rica. Limón, Lomas de Guacimo, Alto la Perla, 1100 m, IV-V. 2013; 4. C. tricolor, female: dorsal view. Costa Rica. San José Province, Cerro de la Muerte, 3362 m, 9³4'00"N 8345'00"W, XI. 2001. 


\section{Description of new taxa}

\section{Chrysina valentini Zubov \& Ivshin sp. $n$.}

zoobank.org: act: 6079BF22-2466-4C42-919F-454C599456F3

Holotype. o", Panama, Ngäbe-Buglé, Nole Duima, Jädeberi 1683 m, 8³1'51"N 8147'55"W, 20.V.2019. Leg. Zaritsky V. \& Gortovanny R. [MNKB], BOLD sample id BC-Ivsh-6212-26 (Fig. 1).

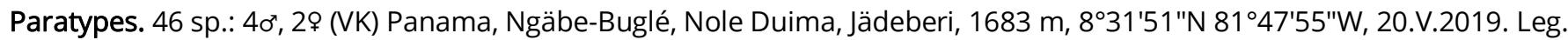

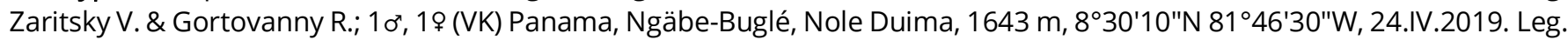
Zaritsky V. \& Gortovanny R.; 30", 3(VK) Panama, Ngäbe-Buglé, Nole Duima, Jädeberi, 1656 m, 8³1'42"N 8147'49"W,

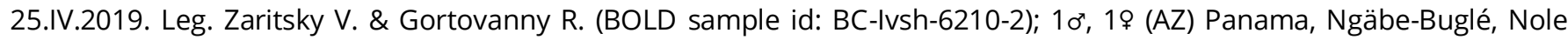
Duima, Jädeberi, 1683 m, 8³1'51"N 8147'55"W, 26.IV.2019. Leg. Zaritsky V. \& Gortovanny R. (BOLD sample id: BC-Ivsh-

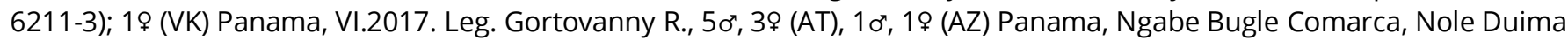

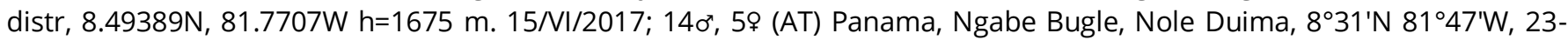
27.05.2018.

\section{Description}

Body length 22-28 mm. Elytra, pronotum and head have shiny golden-silver metallic coloration, abdomen and legs mostly brownish and almost mat. Antennae short, brown, antennal club small, slightly longer in males than in females. Body shape almost oval, twice long as wide. Pronotum slightly trapezoid, approximately 2 times wider than long, measured in its widest part, sides slightly triangular (Fig. 1). Elytra almost 1.2-1.3 times longer than wide. Legs short, hind tibia slightly longer than middle tibia and approximately 1.3 times longer than foreleg tibia. Head, pronotum and elytra glabrous in small rare punctuation. Punctuation slightly stronger on head than on other parts. Clypeus mat, brownish on the sides. Claws long and curvy. Mesosternal process relatively short, thin, brown with golden apex. Elytra with strong stria. Hind femur of males and females slightly thickened comparing to other legs. In comparison with most other Chrysina the foreleg femur bears only two remarkable lateral tubercles in distal part.

\section{Comparative analysis and remarks}

The new species is very close to $C$. optima morphologically but can be easily distinguished by few characters. The foreleg femur of $C$. valentinibears only two distal lateral tubercles in comparison with three tubercles in $C$. optima (Figs $5,6)$. Elytra of $C$. valentini in strong stria almost like in $C$. tricolor, but golden coloration is similar with that in $C$. optima. Elytra of $C$. optima smooth, rarely have weak stria. Mesosternal process of $C$. valentini comparatively thicker than in C. optima and seems bigger (Figs 7, 8).

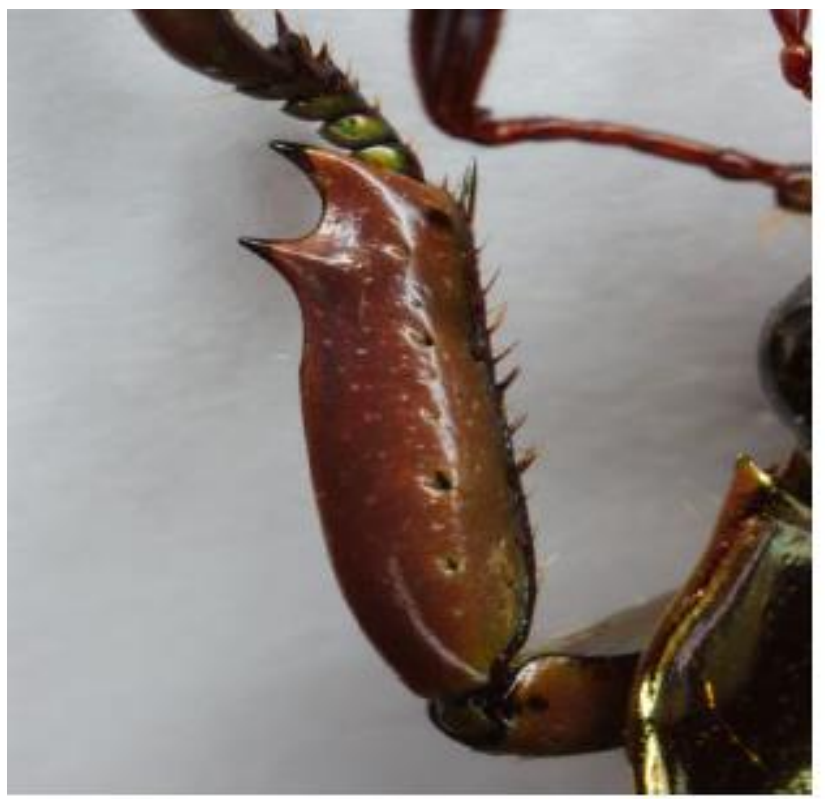

5

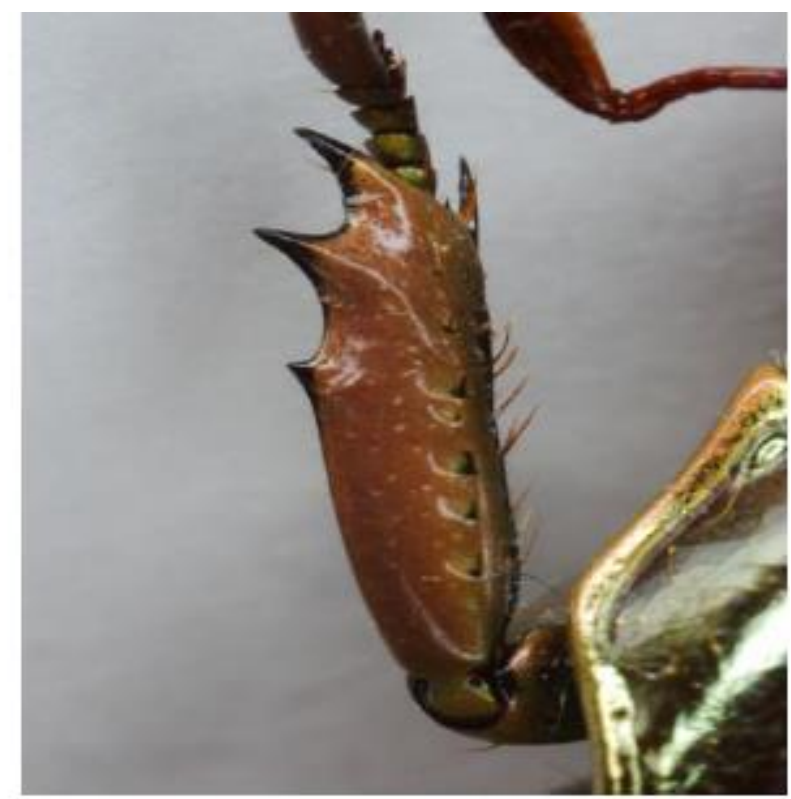

6

Figs. 5-6. Tubercles in distal part of foreleg femur: 5. C. valentini, 6. C. optima 
Erect phallus cornuti of C. valentini and C. optima are shown on Fig. 9-10. Phallus show only slight difference that can't be used for easy diagnostics of the species. Paramere apex of $C$. valentini is slightly more curved. Our technique of genitalia preparation doesn't allow to compare the shape of cornuti reliable enough, but suitable for inspection of their sclerotizations. The picture shows no clear differences between species in these characters. However, this structure can provide significant raise of quality in working with Chrysina in next research.

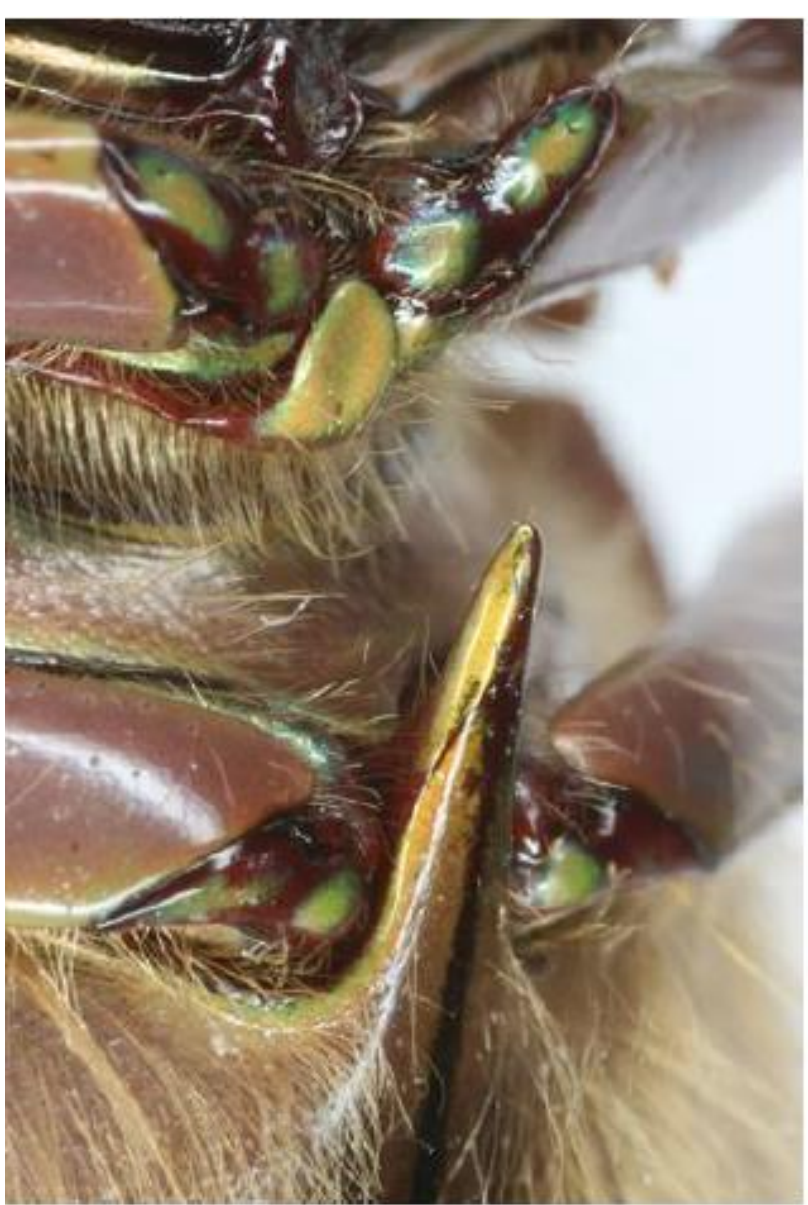

7

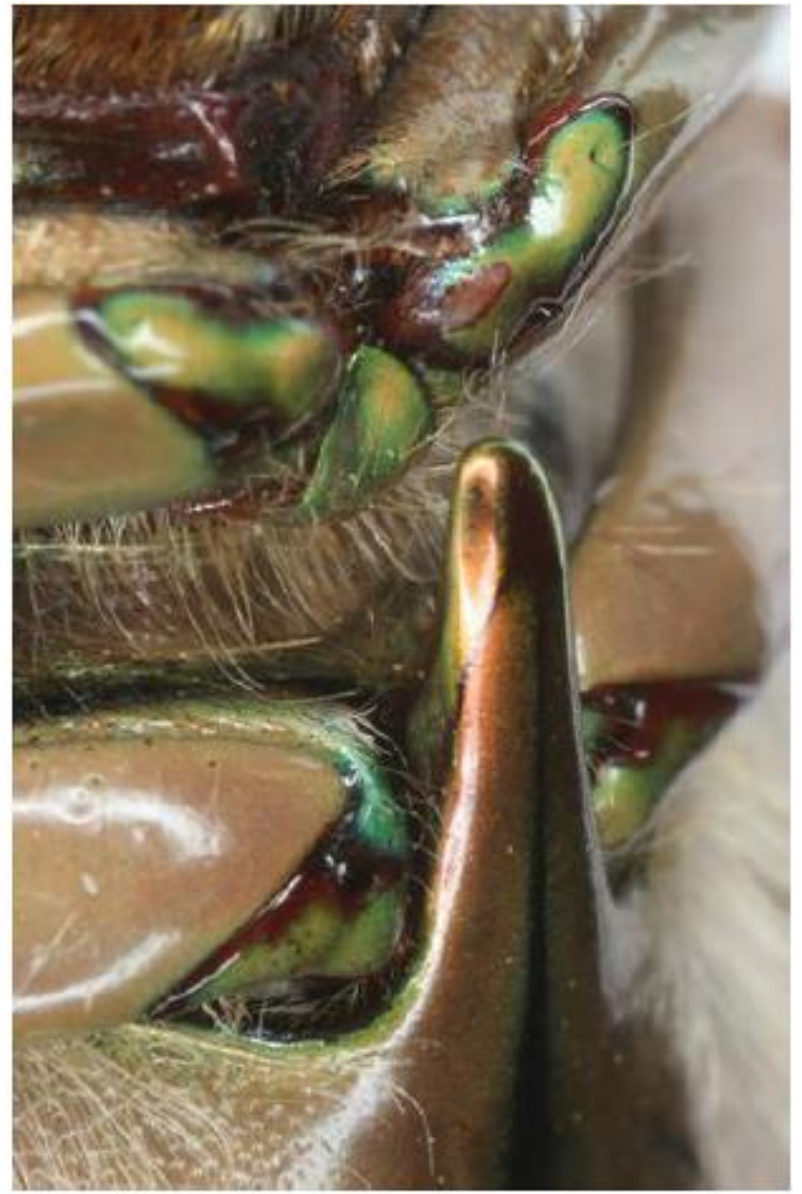

8

Figs. 7-8. Mesosternal process: 7. C. valentini, 8. C. optima

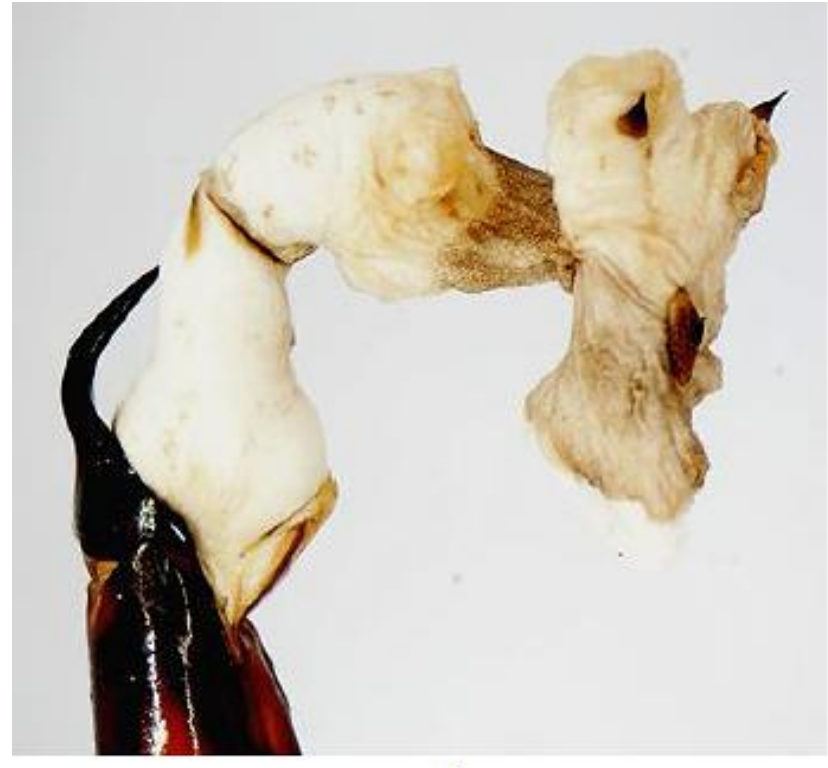

9

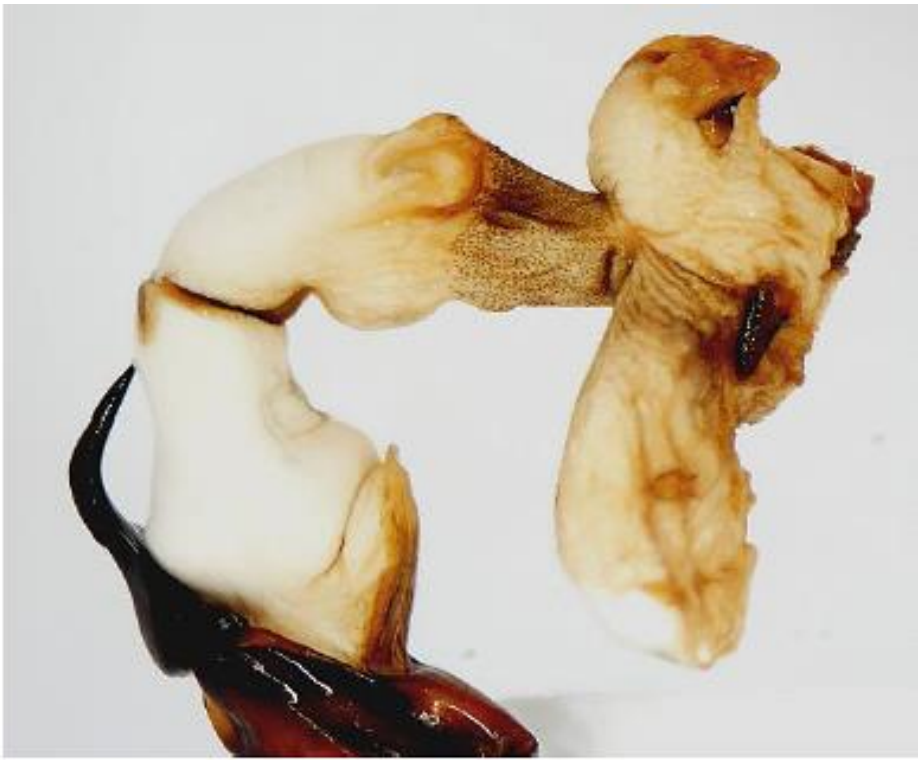

10

Figs. 9-10. Male genitalia, fallus cornuti: 9. C. valentini, 10. C. optima 


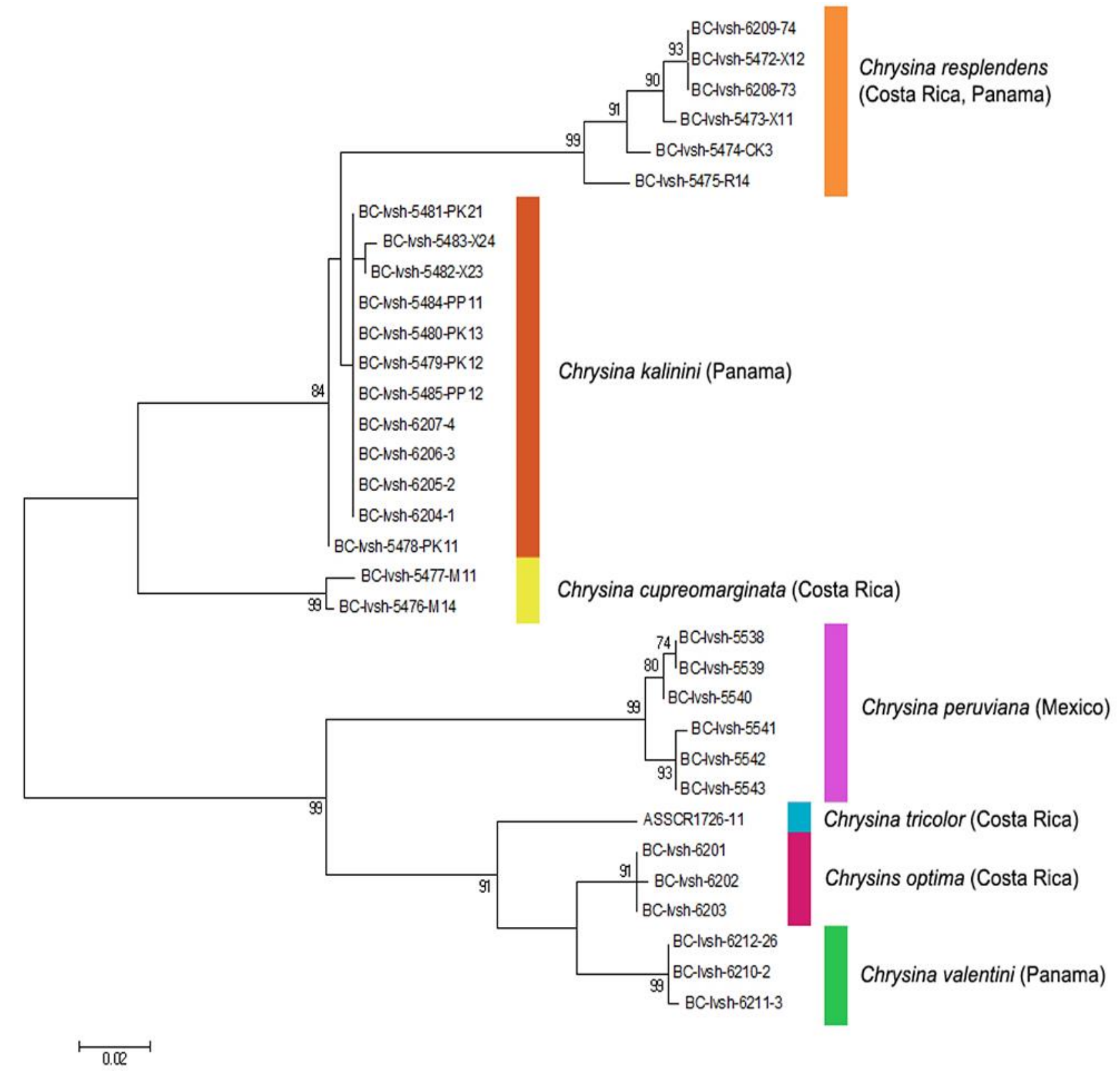

Fig. 11. Maximum likelihood tree of the studied Chrysina. Bootstrap support values of $>70 \%$ are indicated near the branches (500 bootstrap replicates).

In comparison by molecular markers (mtDNA COX I "barcoding region"), C. valentini, C. optima and C. tricolor demonstrated strong divergence. A maximum likelihood tree based on 33 DNA barcode sequences of the new taxa and those of their putative closest relatives in the genus is shown in Fig. 11. Sequences ranged from 500 to 700 bp (sequences shorter than $500 \mathrm{bp}$ were excluded). The number of base substitutions per site from averaging over all sequence pairs between groups was calculated for $C$. valentini and its putative closest relative $C$. optima: $0.034 \pm 0.010$ (the value and standard error estimate, respectively). Analyses were conducted using the Maximum Composite Likelihood model (Tamura, Nei, \& Kumar, 2004). The analysis involved 6 nucleotide sequences. Codon positions included were $1 \mathrm{st}+2 \mathrm{nd}+3 \mathrm{rd}+$ Noncoding. All positions containing gaps and missing data were eliminated. There were a total of 650 positions in the final dataset.

According to the average values of the interspecies pairwise comparisons and the tree topology Chrysina valentini sp.n. reliably differs from C. optima, and molecular divergence of these species is in concordance with their morphology.

Etymology. The species is named after the Russian collector Valentin Alexandrovich Kalinin (Russia, Moscow), who organized the research on this group and who supported a number of research expeditions which were crucial for this study. 


\section{Acknowledgements}

We would like to express our heartfelt appreciation to the following colleagues: our good friend and colleague Pavel Udovichenko for valuable comments, literature and specimens for the study, Valentin Kalinin for support of current research at all stages. This study was supported by the project (№ 0112-2019-0002) «Genetic technology in biology, medicine, agriculture and conservation", on the theme: "Study of the Variability of Autonomous Genetic Elements of Insects and the Development of Genome Instability Markers" (AAAA-A16-116111610180-3).

\section{References}

Bates HW. 1888. Insecta. Coleoptera. Vol. II. Part 2. In: Pectinicornia and Lamellicornia. Salvin O, Godman F du Cane. Editors. Biologia Centrali-Americana. R. H. Porter, London. p 1-432.

Folmer O, Black M, Hoeh W, Lutz R, \& Vrijenhoek R. 1994. DNA primers for amplification of mitochondrial cytochrome c oxidase subunit I from diverse metazoan invertebrates. Molecular Marine Biology and Biotechnology 3: $294-299$.

Hawks DC. 2017. Five new species of Chrysina Kirby (Coleoptera: Scarabaeidae: Rutelinae). Insecta Mundi, April 12, 1-12.

Moore MR, Jameson ML, Garner BH, Audibert C, Smith ABT, \& Seidel M. 2017. Synopsis of the pelidnotine scarabs (Coleoptera, Scarabaeidae, Rutelinae, Rutelini) and annotated catalog of the species and subspecies. ZooKeys 666: 1-349. Doi: https://doi.org/10.3897/zookeys.666.9191

Morón MA. 1990. The Beetles of the World. Volume 10: Rutelini 1. Sciences Nat, Venette.

Ratnasingham S, Hebert PDN. 2007. BOLD: The Barcode of Life Data System (http://www.barcodinglife.org). Molecular Ecology Notes 7: 355-364. Doi: https://doi.org/10.1111/j.1471-8286.2007.01678.x/

Ratcliffe BC. 2002. A checklist of the Scarabaeoidea (Coleoptera) of Panama. Zootaxa 32: 1-48. Doi: https://doi.org/10.11646/zootaxa.32.1.1/

Rothschild W, Jordan K. 1894. Six new species of Plusiotis and one new Anoplostethus. Novitates Zoologicae 1: $504-507$. Doi: https://doi.org/10.5962/bhl.part.24570

Sambrook J, Fritsch EF, Maniatis T. 1989. Molecular cloning: A laboratory manual. Vols. 1-3. 2nd Edition. New York: Cold Spring Harbor Laboratory Press, Cold Spring Harbor.

Tamura K, Nei M, Kumar S. 2004. Prospects for inferring very large phylogenies by using the neighbor-joining method. Proceedings of the National Academy of Sciences (USA) 101: 11030-11035. Doi: https://doi.org/10.1073/pnas.0404206101/

Tamura K, Stecher G, Peterson D, Filipski F, Kumar S. 2013. MEGA6: Molecular Evolutionary Genetics Analysis version 6.0. Molecular Biology and Evolution 30: 2725-2729. Doi: https://doi.org/10.1093/molbev/mst197.

Zubov AS, Ivshin NV, Titarenko AYu, Andrianov BV. 2019. Description of a new species of Chrysina Kirby, 1828 (Coleoptera: Scarabaeidae: Rutelinae) from resplendens group, based on morphological characters and mtDNA COX I molecular marker. Acta Biologica Sibirica 5(1): 71-76. Doi: http://dx.doi.org/10.14258/abs.v5.i1.5194/

\section{Citation:}

Zubov AS, Ivshin NV, Titarenko AYu, Romanov DA. 2019. Description of a new species of Chrysina Kirby, 1828 (Coleoptera: Scarabaeidae: Rutelinae) from optima group, based on morphological characters and mtDNA COX I molecular marker. Acta Biologica Sibirica 5(4): 150-155.

Submitted: 12.11 .2019 . Accepted: 21.12 .2019

cross ref http://dx.doi.org/10.14258/abs.v5.14.7176

(c) 92019 by the authors. Submitted for possible open access publication under the terms and conditions of the Creative Commons Attribution (CC BY) license (http://creativecommons.org/licenses/by/4.0/). 